\title{
Talking to your patients about female genital mutilation
}

This year's Chief Medical Officer report recognises that 'FGM (female genital mutilation) has an impact on the practice of all doctors working within the NHS' (Davies, 2015). In order to identify, treat and support survivors of FGM doctors need to be aware of this practice and its effect on women and girls.

Even doctors who are well informed about the practice can find talking about FGM difficult. We don't want to offend or upset our patients, or appear judgemental by bringing up the topic. As well as trying to find the right words, there is the ever-present concern about having enough time to talk about this sensitively and meaningfully. Often women have never spoken about what has happened to them, yet many have said they expect their GPs to ask them about FGM and help them access support (Norman et al., 2009). Overcoming the barriers to initiating conversations about FGM is likely to be appreciated by our patients, can deepen trusting professional relationships and be very rewarding. This article focuses on how we can talk to patients about FGM. It assumes background knowledge of the practice and its impact on women and girls.

\section{Why ask women about female genital mutilation?}

Discussing FGM with women from FGM-practising communities can make a huge difference to their health and wellbeing. As GPs we are in a great position to ask women about FGM particularly as we are often trusted in situations where other professionals may be seen as intrusive and unwelcome (e.g. social workers, police).

There are three key reasons why you should find out if a woman has undergone FGM:

- It can be relevant to her clinical situation, allowing you assess and treat her appropriately

- Once you identify FGM survivors you can offer support, treat physical and psychological complications of FGM and signpost to other health and community services

- It is an important aspect of child safeguarding - discussing the issue may identify girls at risk and help keep them free from harm

\section{Who should you ask about FGM?}

We should ask all women from FGM-practicing communities about FGM. It is well publicised that FGM is common in Somalia, Ethiopia and Egypt but what about remembering all the other 25+ countries? (UNICEF, 2013) Less well known communities include Iraqi Kurds, Bohra Muslim populations in parts of India and Pakistan, and Muslim communities in Malaysia and Indonesia.

If you are not sure if a woman is from an FGM-practicing community, do not let it put you off bringing the subject up. Just ask a simple and neutral question to find out, e.g.

- Around the world some communities practice female circumcision or cutting. Does this happen in your community? 


\section{When should you ask women about FGM?}

There are some clinical situations when asking about FGM is a must. Think about FGM when you see women from FGM-practicing communities with:

- Menstrual problems - dysmenorrhoea, menorrhagia, primary amenorrhoea

- During pregnancy, especially for women with Type 3 FGM so deinfibulation can be discussed

- Depression/ post traumatic stress disorder symptoms

- Sexual difficulties - dyspareunia, difficulty with penetrative sex, lack of desire

- Recurrent urinary tract infections, urinary incontinence

- Perineal pain or infection

- Prior to vaginal/speculum examination

\section{How do you bring up the subject of FGM?}

Mutilation is not a neutral word. Some women find the term FGM offensive or difficult to hear as it can make them feel judged and abnormal. The term "female circumcision" is controversial because it can imply the procedure is equivalent to male circumcision and so minimises associated harm and mortality. However some survivors of FGM prefer this term and you should respect that when talking to them. Mirror your patient's language.

If a woman does not know what you mean when you ask about FGM try alternative phrases:

- Have you been cut/circumcised/closed?

- Have you ever had any operations or been cut on your vagina/genitals/down below?

- Has anything ever been done to you to change your appearance 'down below'?

Taking a moment to signpost why you are asking means your questions should not come across as irrelevant or intrusive, e.g.

- Sometimes your symptoms can be caused by something called FGM or female circumcision

- It's important to know if a pregnant woman has had anything done which might affect her when she gives birth. Such as being cut or closed down below.

- Before I examine you I just need to check if you've ever had any operations or been cut?

If FGM is not directly relevant to the consultation, then just like asking about smoking or contraception, you should use your clinical judgement about when to bring it up. Sometimes you just need to bite the bullet and ask! Asking non-judgmental direct questions will help you bring up FGM in a sensitive way, e.g.

- I know that FGM or female circumcision is common in Somalia. It's important for your health to know if this has happened to you... have you had FGM done or been circumcised or closed?

- Something we routinely ask women from Sierra Leone is whether they have been cut or circumcised. Is this something that has happened to you? 


\section{Once you've identified a woman has had FGM what else should you ask her?}

As always start with exploratory questions such as Do you think the circumcision has caused you any problems? Do you ever think about it?

Ask about possible complications of FGM (see main article about FGM in this issue and point 3 above). Don't forget to ask about psychological harm such as flashbacks, nightmares or body image issues (FORWARD, 2012). You may wish to ask the woman if she remembers being cut, how old she was and where it was done.

It is vital to find out if she has any daughters and assess their risk - see point 7 below.

\section{If a woman has had FGM done what should you do?}

Sometimes listening is all you can and need to do. Don't try to fix what has happened; you can't. Give the woman space to acknowledge what's happened to her - a form of violence which for some results in life-long emotional scars.

Many FGM survivors do not know what has been done to them and may find it helpful to have this explained after an examination. Offer women referral to specialist FGM clinics and tell them about community organisations that provide support.

Explain FGM is illegal in the UK - even if a girl/teenager is taken abroad to have this done. You may wish to offer the woman an 'FGM passport' (a small leaflet produced by the Home Office) which she can use to show others FGM is illegal in the UK (HM Government, 2015).

Of course you should document your consultation as you would any other. Since October 2015 it is mandatory for GPs in England to record specific data about FGM. This data is then extracted to the Health and Social Care Information Centre. As this data contains patientidentifiable information, serious concerns have been raised about this process breaching patient confidentiality and some practices are refusing to pass on this information. Speak to your practice about how they are addressing this issue, whether they have an FGM template and which codes to use.

\section{If you are worried that an under 18 year-old has had or is at risk of having FGM done what should you do?}

If a woman with FGM has daughters you should find out if they have had FGM done and if she has plans to have them cut. It is important to find out about pressures from others. Is she concerned that others in her family or community want her daughters or other family members to have FGM done? If you have any concerns about a girl being at risk of FGM you must deal with it as you would any other child safeguarding case: talk to your trainer, speak to social services urgently, explain this to the woman (unless you have concerns that doing so would put the girl(s) in question at more risk).

Since October 2015, healthcare professionals in England and Wales have a mandatory legal duty to report FGM in any under 18 year-old to the police (Serious Crime Act 2015). You should ring 101 yourself, ideally within 24 hours of identifying a girl with FGM. It is 
important to take the time to explain to the child's parents that you are going to contact the police and that you have a legal duty to so.

\section{Who can you ask for advice about FGM?}

If you don't know where your nearest FGM clinic is your local gynaecology team should be able to signpost you. If you are not sure whether a particular case is a child safeguarding issue then speak to your social service team urgently. As with any child safety concerns, don't ignore any worries and act promptly.

\section{References and further information}

- Davies, S.C. (2015). Annual Report of the Chief Medical Officer, 2014, The Health of the 51\%: Women. London: Department of Health

- Health Education England e-learning to improve awareness and understanding of FGM; Available from: http://www.e-lfh.org.uk/programmes/female-genitalmutilation/

- Foundation for Women's Health, Research and Development (FORWARD). (2012). Female Genital Mutilation: Frequently Asked Questions: A Campaigner's guide for Young People

- HM Government. (2015). A Statement Opposing Female Genital Mutilation

- Norman, K., Hemmings, J., Hussein, E., Ootoo-Oyortey, N. (2009). FGM is always with us. Experiences, Perceptions and Beliefs of Women Affected by Female Genital Mutilation in London.

- UNICEF. (2013). Female Genital Mutilation/Cutting: A statistical overview and exploration of the dynamics of change 